\title{
Educação em saúde - estratégia de cuidado integral e multiprofissional para gestantes
}

Maria Luiza Alves Araújo*, Ariany Paula Medeiros*, Sara Zuculin**, Evelin Gonçalves Souza**, Paula Ferreira Barros**, Talita Boaventura***, Patrícia Helena Costa Mendes****, Ana Paula Ferreira Maciel*****, Mariano Fagundes Neto******

* Acadêmicas do PET-Saúde e do Curso de Odontologia da Universidade Estadual de Montes Claros, Unimontes

** Acadêmicas do PET-Saúde e do Curso de Medicina da Universidade Estadual de Montes Claros, Unimontes

*** Acadêmica do PET-Saúde e do Curso de Educação Física da Universidade Estadual de Montes Claros, Unimontes

**** Preceptora do PET-Saúde da Unimontes e Cirurgiã-dentista de Família da Secretaria Municipal de Saúde de Montes Claros, Minas Gerais

***** Preceptora do PET-Saúde da Unimontes e Enfermeira de Família da Secretaria Municipal de Saúde de Montes Claros, Minas Gerais

****** Preceptor do PET-Saúde da Unimontes e Médico de Família da Secretaria Municipal de Saúde de Montes Claros, Minas Gerais

\section{RESUMO}

O Programa de Educação pelo Trabalho para a Saúde (PET-Saúde) objetiva iniciar os estudantes das graduações em saúde no trabalho da Estratégia Saúde da Família, oportunizando a vivência das ações desenvolvidas na atenção básica à saúde. Dentre estas atividades, destaca-se a educação em saúde que integra o saber científico com o popular. Um dos grupos prioritários que deve ser alvo da atividade de educação em saúde na Estratégia Saúde da Família é o grupo de gestantes, por se tratar de um período em que a mulher está mais susceptível a receber informações e modificar o comportamento. O objetivo deste trabalho é expor uma experiência de cuidado integral e multiprofissional, utilizando-se a educação em saúde como ferramenta para a adoção de novos hábitos em saúde por parte de um grupo de gestantes assistidas por uma equipe da Estratégia Saúde da Família. O projeto foi realizado no mês de janeiro de 2011 na Equipe de Saúde da Família Independência III, localizado no município de Montes Claros/MG, tendo como agentes facilitadores os acadêmicos da área da saúde da Universidade Estadual de Montes Claros, participantes do PET-Saúde, bem como os profissio- nais de saúde, que integram a equipe, sendo também preceptores do programa. O curso foi estruturado em sete oficinas, abordando temas chaves para as gestantes. Esse projeto foi de grande importância tanto para as gestantes, que avaliaram de forma positiva a atividade, quanto para os acadêmicos, que puderam adquirir conhecimentos de forma crítica e multidisciplinar.

\section{DESCRITORES}

Gestantes. Educação em saúde. Atenção primária à saúde.

$\mathbf{0}$ Ministério da Saúde e da Educação tem incentivado a aproximação das universidades e dos serviços de saúde prestados à população, através da implantação de projetos como o Programa de Educação pelo Trabalho para a Saúde (PET-Saúde), instituído pela Portaria Interministerial MEC-MS n ${ }^{\circ} 1.802$, de 26 de agosto de 2008 dos Ministérios da Educação e da Saúde. Este programa destina-se a fomentar grupos de aprendizagem tutorial na Estratégia Saúde da Família, visando o fortalecimento da atenção básica em saúde, de acordo com os princípios e as necessi- 
dades do Sistema Único de Saúde (SUS)..$^{3,10}$

O PET-Saúde tem como fio condutor a integração ensino-serviço-comunidade, caracterizando-se como instrumento para qualificação em serviço dos profissionais da saúde, bem como de iniciação ao trabalho e vivências dirigidos aos estudantes das graduações em saúde. Desta forma, o PET-Saúde estimula a formação de profissionais de saúde com perfil adequado às necessidades e às políticas públicas de saúde do país e, consequentemente, almeja a satisfação dos usuários do SUS. ${ }^{10}$

A atenção básica, cujo eixo reorientador é a Estratégia Saúde da Família, percebe a prática sanitária como uma combinação de ações de promoção de saúde, prevenção das doenças e enfermidades e atenção curativa. Além disso, preconiza o cuidado integral ao indivíduo, à família e à comunidade na perspectiva interdisciplinar e multiprofissional. ${ }^{2}$

No que diz respeito à organização dos serviços e das práticas de saúde, a integralidade caracteriza-se pela assimilação das práticas preventivas e das práticas assistenciais por um mesmo serviço. De acordo com o princípio da integralidade, a abordagem do profissional de saúde não deve se restringir à assistência curativa, buscando dimensionar fatores de risco à saúde e, por conseguinte, a execução de ações preventivas, a exemplo da educação para a saúde. ${ }^{1}$

A educação em saúde é o campo de prática e conhecimento do setor saúde que tem se ocupado mais diretamente com a criação de vínculos entre a ação médica e o pensar e fazer cotidiano da população. Constitui-se em um processo de trocas de saberes e experiências entre a população como um todo, incluindo usuários, profissionais e gestores de saúde. ${ }^{14}$ Esta prática visa à prevenção de doenças, promove a autonomia dos sujeitos envolvidos, tornando-os sujeitos ativos e transformadores de sua própria vida ou até mesmo da sua sociedade. ${ }^{16}$

A integralidade no cuidado de pessoas, grupos e coletividade entende o usuário como sujeito histórico, social e político, articulado ao seu contexto familiar, ao meio ambiente e à sociedade na qual se insere. Neste cenário, evidencia-se a importância das ações de educação em saúde como estratégia integradora de um saber coletivo que traduza no indivíduo sua autonomia e emancipação. ${ }^{6,13}$

Educar para a saúde implica dar prioridade a intervenções preventivas e promocionais, em espaços coletivos, como por exemplo, os grupos educativos.

Um dos grupos prioritários que deve ser alvo da atividade de educação em saúde na Estratégia Saú- de da Família é o grupo de gestantes. A gestação caracteriza-se por um período em que a mulher está mais susceptível a receber informações e modificar o comportamento. A gestação representa um momento especial na vida da mulher, no qual a sensação de tornar-se mãe confunde-se muitas vezes com incertezas, medos e inseguranças. É um evento biossocial, pois está cercado de valores culturais, sociais

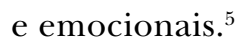

A carência de informações ou informações inadequadas sobre o parto, o medo do desconhecido, bem como os cuidados a serem prestados ao recém-nascido nos primeiros dias são fatores comuns de tensão da gestante, que influenciam negativamente durante todo o processo. É de competência da equipe de saúde acolher a gestante e a família desde o primeiro contato com a unidade de saúde. ${ }^{11}$

Contudo, o que se pode notar, atualmente, em várias rotinas de serviços de saúde da atenção básica é a pouca prática de atividades de educação em saúde voltadas ao coletivo, dando-se prioridade, por diversos motivos, aos atendimentos individuais, limitados ao espaço físico de uma sala de atendimento. ${ }^{12}$ No âmbito da assistência pré-natal, o processo educativo restringe-se ao fornecimento de informações sobre alguns aspectos relacionados à gravidez, parto e cuidados com o bebê durante a consulta realizada pelo profissional de saúde. É imprescindível, assim, que todas as categorias de profissionais de saúde que atuam na Estratégia Saúde da Família considerem a gestação como um momento ímpar para a realização de ações educativas coletivas.

O objetivo deste trabalho é, a partir da descrição de um relato de experiência, apresentar um projeto de educação em saúde direcionado às gestantes de uma comunidade norte-mineira desenvolvido por acadêmicos integrantes do PET-Saúde dos cursos de Odontologia, Medicina, Enfermagem e Educação Física da Universidade Estadual de Montes Claros e por profissionais médico, enfermeira, e cirurgiã-dentista da Secretaria Municipal de Saúde de Montes Claros/MG, que atuam como preceptores do programa. Este estudo procura expor uma experiência de cuidado integral e multiprofissional, utilizando-se a educação em saúde como ferramenta para a adoção de novos hábitos e condutas em saúde por parte de um grupo de gestantes assistidas por uma equipe da Estratégia Saúde da Família.

\section{MATERIAL E MÉTODOS}

Utilizando-se a ideologia da Educação Popular proposta por Paulo Freire, foi realizado no mês de 
janeiro de 2011 na Equipe de Saúde da Família Independência IIII no bairro Independência, localizado no município de Montes Claros/MG, o "I Curso para Gestantes" tendo como agentes facilitadores os acadêmicos de Odontologia, Medicina, Enfermagem e Educação Física da Universidade Estadual de Montes Claros, todos participantes do PET-Saúde, bem como os profissionais de saúde médico, enfermeira e cirurgiã-dentista, que integram a equipe, sendo também preceptores do programa.

A Educação Popular em Saúde realiza ações que envolvem as dimensões do diálogo, do respeito e da valorização do saber popular, sendo considerada um instrumento de construção para uma saúde mais integral e adequada à vida da população, representando uma prática de saúde onde não há domínio de um saber sobre outro. ${ }^{8}$

O curso para gestantes foi organizado em encontros semanais, realizados as terças e quintas-feiras à tarde na unidade de saúde da família. O curso foi estruturado em sete oficinas, com tempo de duração previsto de cinquenta minutos cada, não excedendo o limite de vinte participantes.

Para o desenvolvimento deste projeto, houve a participação ativa das mulheres, valorizando o diálogo, favorecendo o reconhecimento das usuárias enquanto sujeitos portadores de saberes sobre o processo saúde-doença-cuidado, evitando-se o monólogo de palestras em que se busca apenas transferir conhecimentos.

Para cumprir com o objetivo proposto, as oficinas abordaram os seguintes temas, conforme mostra a Tabela 1.

Na primeira oficina, houve a apresentação dos temas pré-selecionados, estruturados de acordo com a Linha do Cuidado à Gestante, Puérpera e ao Re-

Tabela 1 - Temas das oficinas do curso de gestantes.

\begin{tabular}{|l|l|}
\hline Oficina 01 & $\begin{array}{l}\text { Apresentação do curso, expectativas,varal de } \\
\text { idéias e discussão sobre direitos e deveres } \\
\text { durante a pré-concepção, pré-natal, parto e } \\
\text { puerpério }\end{array}$ \\
\hline Oficina 02 & Cuidados alimentares durante a gestação \\
\hline Oficina 03 & $\begin{array}{l}\text { Cuidados com as mamas e importância da } \\
\text { amamentação }\end{array}$ \\
\hline Oficina 04 & Sinais de parto \\
\hline Oficina 05 & $\begin{array}{l}\text { Saúde bucal na gestação, cuidados com o } \\
\text { recém-nascido e acompanhamento da criança }\end{array}$ \\
\hline Oficina 06 & Sexualidade e planejamento familiar \\
\hline Oficina 07 & $\begin{array}{l}\text { Técnicas de relaxamento para gestantes e } \\
\text { confraternização de encerramento }\end{array}$ \\
\hline
\end{tabular}

cém-Nascido da Secretaria Estadual de Saúde de Minas Gerais, enfatizando a importância do conhecimento destes para um bom transcurso da gravidez. Realizou-se também um Varal de Idéias em que as gestantes propuseram temas para serem abordados. Posteriormente, foi realizada uma dinâmica visando à integração do grupo. Nesse momento, as gestantes expuseram suas expectativas relacionadas ao curso, que incluíram adquirir conhecimentos sobre uma gestação saudável, tipos de parto e cuidados com o recém-nascido. A abordagem dos direitos e deveres da gestante durante a pré-concepção, o pré-natal, o parto e o puerpério estimularam discussões relacionadas ao parto humanizado com direito à acompanhante na sala de parto e direito trabalhistas relacionados à maternidade.

A oficina 02, que abordou o tema alimentação e ganho de peso na gestação, foi bastante dinâmica havendo grande participação das presentes. Foram unânimes dúvidas quanto à dieta ideal de uma grávida. Assim, cada participante relatou suas experiências com hábitos alimentares, expondo sinais e sintomas percebidos com cada alimento e alterações ao longo desse período.

A oficina 03 teve como foco os cuidados com as mamas durante e após a gestação. Foi enfatizada a importância da amamentação para o recém-nascido, o tempo ideal de duração do aleitamento materno e a maneira correta de a criança sugar as mamas maternas. Foi exibido um vídeo a fim de demonstrar como deve ser feita a "pega" correta pela criança.

Durante a oficina 04, as participantes foram questionadas quanto aos principais temores sobre o parto, principalmente, sobre o parto normal. Além disso, foi indagado às grávidas, que já eram mães, como havia sido os partos anteriores e se havia ocorrido alguma intercorrência. Depois do relato das experiências e receios das mesmas, discutiu-se sobre as vantagens do parto normal e sobre os sinais indicativos do trabalho de parto.

$\mathrm{Na}$ oficina 05 , inicialmente, as acadêmicas de odontologia falaram sobre os cuidados com a saúde bucal do recém-nascido e da criança em seus primeiros anos de vida, usando cartazes explicativos e esclareceram sobre os mitos relacionados ao tratamento odontológico durante a gravidez. Percebeu-se que muitas gestantes ainda temiam o uso do anestésico local e da tomada de radiografias dentárias. Posteriormente, foram abordados os principais cuidados com o recém-nascido, ressaltando a maneira correta de dar banho no mesmo através da exibição de um vídeo. 
Além desse tópico, discutiu-se também sobre cuidados com o cordão umbilical, icterícia neonatal fisiológica, vacinas, cólicas, aparência e consistência das fezes do recém-nascido e o teste do pezinho. Depois, houve a distribuição de folders com o resumo dos principais cuidados abordados.

$\mathrm{Na}$ oficina 06 , utilizando-se desenhos ilustrativos, abordou-se a sexualidade durante a gravidez, com demonstração de posições que permitem a realização de relação sexual sem incômodo em cada fase da gestação. Observou-se bastante curiosidade acerca do assunto. Posteriormente, foi abordado o planejamento familiar com a explicação dos métodos contraceptivos. Para efeito didático, foram usados cartazes ilustrativos e apresentação de alguns dos métodos abordados.

Durante a sétima e última oficina, foi abordado, pela acadêmica de educação física, técnicas de relaxamento corporal utilizando bolinhas de borracha. As gestantes ficaram satisfeitas com a facilidade das técnicas e com a possibilidade de realização em casa. Posteriormente, foi realizado um bingo que teve como brinde uma cesta com produtos para o futuro recém-nascido e, ao final, houve a entrega para cada participante de um certificado com os dizeres "Pronta para ser Mãe".

\section{RESULTADOS E DISCUSSÃO}

A atenção pré-natal e puerperal qualificada e humanizada acontece por meio da incorporação de condutas acolhedoras e sem intervenções desnecessárias, de fácil acesso aos serviços de saúde de qualidade, com ações que integrem todos os níveis da atenção:

- promoção,

- prevenção e

- assistência à saúde da gestante e do recém-nascido. ${ }^{17}$

Dentre estas ações, a realização de ações educativas no decorrer de todas as etapas do ciclo grávidopuerperal faz-se muito importante. ${ }^{7}$ Para isso, os profissionais de saúde devem assumir a postura de educadores que compartilham saberes, buscando devolver à mulher sua autoconfiança para viver a gestação, o parto e o puerpério. ${ }^{11}$

Para que o curso de gestantes obtivesse êxito, foi preciso conhecer e ouvir cada participante, pois, a partir de suas necessidades e vivências, foi possível avaliar as dificuldades e encontrar a potencialidade do grupo. Portanto, saber ouvir representa habilidade fundamental nos processos de educação em saúde.

As ações educativas em saúde possibilitam um mo- mento de intenso aprendizado e mediante as atividades grupais podem-se constituir um método efetivo e simples de se intervir a favor da melhoria da qualidade de vida durante a gestação e no puerpério. O grupo de gestantes é um meio de promover a educação em saúde, com o objetivo de preparar a mulher e sua família para o processo gestacional, expressando o que sentem e sanando suas dúvidas relativas ao momento que estão vivenciando. ${ }^{15}$ Assim, entende-se que o contexto grupal desenvolve naturalmente um espaço para o movimento da promoção da saúde através de um processo de ensinar-aprender.

Informações sobre as diferentes vivências foram trocadas entre as mulheres, estudantes e os profissionais de saúde. Essa possibilidade de intercâmbio de experiências e conhecimentos foi considerada a melhor forma de promover a compreensão de vários aspectos relacionados ao processo de gestação.

A partir da horizontalizarão da relação existente entre profissional de saúde e usuário, o que facilita a expressão individual e coletiva das necessidades e expectativas, pode-se definir os principais temas a serem discutidos:

- direitos e deveres durante pré-concepção, prénatal, parto e puerpério;

- alimentação na gestação;

- saúde bucal na gestação;

- cuidados com as mamas e importância da amamentação;

- sinais de parto;

- cuidados com o recém-nascido e acompanhamento da criança;

- planejamento familiar e sexualidade.

Sentindo-se pertencentes a um grupo de pessoas que vivenciavam o mesmo fenômeno: a gravidez, as gestantes puderam esclarecer as suas dúvidas, falar sobre os medos e dificuldades e adquirir novos conhecimentos acerca do cuidado com a sua saúde e do recém-nascido.

Assim, é possível compreender que a educação em saúde está intimamente relacionada às ações cuidadoras. Isso remete à dupla identidade dos profissionais de saúde - a de educador e a de trabalhador de saúde. Essa duplicidade mostra que a educação ocupa lugar central no trabalho em saúde e, muitas vezes, é o que o torna viável.

\footnotetext{
"Não é possível pensar a saúde sem, simultaneamente, pensar a educação e as relações existentes entre ambas". ${ }^{9}$
} 
Destaca-se, ainda, a importância da abordagem multiprofissional à gestante. A gama de situações vivenciadas nessa fase evidencia a necessidade de uma atuação em diversos aspectos como:

- a história pessoal,

- os antecedentes ginecológicos e obstétricos,

- o momento histórico da gravidez,

- as características sociais, culturais e econômicas vigentes

- e qualidade da assistência.

A assistência integral deve ser capaz de proporcionar à mulher e ao concepto um período satisfatório de bem-estar, visando o fortalecimento do vínculo mãe-feto. ${ }^{4}$

A iniciação ao trabalho na Estratégia Saúde da Família proposta pelo PET-Saúde propõe unir os conhecimentos e experiências de todas as categorias profissionais e assim compreender o processo saúde/ doença, conhecer as causas e consequências e encontrar os caminhos para resolução dos problemas identificados. Certamente, o trabalho interdisciplinar realizado no PET-Saúde encontra caminhos alternativos para intervir e modificar, enfrentar novas situações, novas formas de entender e de ver nossa realidade concreta.

O curso de gestantes desenvolvido pelas acadêmicas da Universidade Estadual de Montes Claros foi produtivo, uma vez que o período gestacional despertou nas participantes interesse maior acerca dos assuntos abordados. A participação ativa das gestantes contribuiu para uma troca de aprendizados, possibilitando o bom resultado dessa iniciativa de educação em saúde.

As gestantes avaliaram de forma positiva a atividade, pois a partir das informações recebidas e das metodologias aplicadas, sentiram-se mais seguras e confiantes para desempenhar seu papel de mãe.

Para as acadêmicas participantes do PET-Saúde, essa atividade foi enriquecedora, pois contribuiu para a aquisição do conhecimento de forma crítica, a partir da vivência de uma experiência de trabalho em equipe, percebendo a integração e os limites de atuação de cada categoria profissional, valorizando as especificidades de cada profissão.

\section{CONClusões}

O curso de gestantes construiu um espaço de trocas de experiências, saberes e vivências que levaram à construção e à reconstrução do conhecimento a partir de um processo de identificação entre os atores envol- vidos. Pode-se afirmar que houve, nesse processo, uma construção de conhecimento compartilhado, que leva as mulheres a fazerem escolhas conscientes sobre suas condutas em relação à gestação e cuidados com o recém-nascido. Acredita-se que estratégias como esta auxiliam na melhoria da qualidade da assistência prestada à mulher durante o período gestacional.

A experiência de uma atividade educativa elaborada na perspectiva do cuidado integral e multiprofissional contribuiu para a formação dos acadêmicos da Universidade Estadual de Montes Claros, integrantes do PET-Saúde, uma vez que vivenciaram e superaram os desafios do trabalho em equipe, apoderandose da dinâmica de trabalho da Estratégia Saúde da Família.

\section{ABSTRACT}

Health education - strategy of integral and multiprofessional care for pregnant women

The Education Program through Health Work ("PET-Saúde") aims at initiating undergraduate students of health into Family Health Strategy work, allowing them to experience the actions conducted in primary care. Among these activities, there is health education that combines scientific with popular knowledge. One of the priority groups that should be targeted for health education activities in the Family Health Strategy is that of pregnant women, considering that this is a period in which a woman is more likely to receive information and to modify her behavior. The objective of this study was to present an experience of integral and multiprofessional care, using health education as a tool for adopting new health habits by a group of pregnant women assisted by a team from the Family Health Strategy. The project was conducted in January 2011 by the Independence III Family Health Team, located in Montes Claros, MG. The team included students from the health field at the State University of Montes Claros as facilitators, participants in the "PET-Saúde" Program, and health professionals, who were also instructors of the program. The course was structured around seven workshops, and addressed key issues for pregnant women. This project was of great importance not only for the pregnant women, who evaluated the activity positively, but also for the students, who were able to acquire critical and multidisciplinary knowledge.

\section{DESCRIPTORS}

Pregnant women. Health education. Primary health care. 


\section{REFERÊNCIAS}

1 Alves VS. Um modelo de educação em saúde para o Programa Saúde da Família: pela integralidade da atenção e reorientação do modelo assistencial. Interface-Comunic Saúde Educ. 2005; 9(16):39-52.

2 Brasil. Governo do Estado de São Paulo. Manual para operacionalização das ações educativas no SUS. São Paulo. 2001.

3 Datasus [homepage]. Brasília, DF: Ministério da Saúde [acessado em 09 fev. 2011]. [3 telas]. Disponível em: < http://www. datasus.gov.br/cns $>$.

4 Falcone, VM et al. Atuação multiprofissional e a saúde mental das gestantes. Rev Saúde Pública 2005; 39(4):612-8.

5 Ferreira ANS. Parto cesariano: opinião de mulheres. Textura. 2008;3(2):82-95.

6 Machado MFAS et al. Integralidade, formação de saúde, educação em saúde e as propostas do SUS - uma revisão conceitual. Ciênc Saúde Coletiva. 2007;12(2):335-342.

7 Moura ERF, Rodrigues MSP. Comunicação e informação em saúde no pré-natal. Interface. 2003;7(13):109-118.

8 Neto PJl, Batista PSS. Projeto educação em saúde na atenção a gestantes e puérperas. Anais X Encontro de Extensão. 2008. 9p. Paraíba. Brasil.

9 Penna CMM, Santos RV. A educação em saúde como estratégia para o cuidado à gestante, puérpera e ao recém-nascido. Texto Contexto Enferm. 2009;18(4): 652-60.

10 Portal Saúde [homepage]. Brasília, DF: Ministério da Saúde [acessado em 09 fev. 2011]. [2 telas]. Disponível em: < www. saude.gov.br/sgtes/petsaude > .

11 Rios CTF, Vieira NFC. Ações educativas no pré-natal: reflexão sobre a consulta de enfermagem como um espaço para educação em saúde. Ciênc. Saúde Coletiva. 2007; 12(2):477-486.

12 Rolim M, Moreira T, Viana G. Course for pregnant mothers: educational action from co-responsibility standpoint. A descriptive study. Online Braz J Nursing. [acesso 10 Fev 2011]. 2006. Disponível em: < http://www.objnursing.uff.br/index. $\mathrm{php} /$ nursing/article/view/595/140 > .

13 Santana MCCP. Aleitamento materno em prematuros: atuação fonoaudiológica baseada nos pressupostos da educação para promoção da saúde. Ciênc. Saúde Coletiva. 2010;15(2):411417.

14 Schall VT, Struchiner M. Educação em saúde: novas perspectivas. Cad. Saúde Pública. 1999;15(2):4-6.

15 Silveira IP et al. Ação educativa à gestante fundamentada na promoção da saúde: uma reflexão. Esc. Anna Nery Ver Enferm. 2005; 9(3):451-458.

16 Vasconcelos EM. Educação popular: de uma prática alternativa a uma estratégia de gestão participativa das políticas de saúde. Physis: Rev. Saúde Coletiva. 2004;14(1):67- 83.

17 Zampieri MFM et al. Processo educativo com gestantes e casais grávidos: possibilidade para transformação e reflexão da realidade. Texto Contexto - Enferm. 2010; 19(4):719-727.

Recebido em 07/07/2011 Aceito em 25/07/2011 